\title{
RELACIÓN DE REVISORES DEL AÑO 2014
}

\author{
LIST OF REVIEWERS 2014
}

El Comité Editor de la Revista Peruana de Medicina Experimental y Salud Pública (RPMESP) agradece a cada uno de los destacados profesionales, expertos en sus respectivas áreas, quienes han colaborado de forma desinteresada y con celeridad en la revisión de los manuscritos que les fueron enviados para su evaluación.

Cuando llega un artículo a la RPMESP, el Comité Editor, luego de revisar que cumpla con los requisitos estipulados en las normas para los autores, evalúa la pertinencia de su publicación en la revista, que no tenga conflictos éticos en publicación y que su calidad sea adecuada para la revista, decide enviarlo a dos o más revisores en el caso de artículos originales (incluyendo breves) y uno o más en reportes de casos, revisiones, simposios, entre otros.

La selección de revisores se realiza teniendo en cuenta la lista de revisores que cada año va en aumento; así mismo, se realiza una búsqueda de investigadores en Pubmed y el Google Académico, que hayan publicado artículos similares al que está en evaluación, esta búsqueda se inicia explorando artículos aparecidos en estas bases o a partir de las citas que tiene el estudio; posteriormente, se identifican a los investigadores y se evalúa si sus publicaciones son relacionadas con el tema de interés; si así lo es, y habla español (lo deducimos por su filiación o si ha publicado en español) se busca su correo electrónico y es contactado por esa vía. Este mecanismo de selección de revisores ha permitido que nuestro proceso de revisión sea más rápido, de mayor calidad, y con mayor participación de investigadores extranjeros que nacionales.

\author{
Abarca Villaseca, Katia \\ Abeyá Gilardon, Enrique \\ Acedo Félix, Evelia \\ Acosta Peñaloza, Jesús Antonio \\ Agrela, Irma \\ Antiporta, Daniel \\ Aracena Álvarez, Marcela \\ Arboix Damunt, Adriá \\ Arévalo Flores, Jorge Martín \\ Argente, Jesús \\ Arroyo Hernández, Hugo \\ Arrufat Gallén, Vita \\ Asunción Iborra, María \\ Ayuga Téllez, Esperanza \\ Ayuso Álvarez, Ana \\ Bambarén Alatrista, Celso \\ Barrera Pérez, Mario Antonio \\ Barriga Doy, Guillermo \\ Benites Zapata, Vicente \\ Bernardo, Jose F.
}

Best Bandenay, Pablo Yván

Bonilla Sepúlveda, Óscar Alejandro

Botella, Juan

Bravo Luna, Marta

Burstein Alva, Zuño

Bustamante Chávez, Inés

Cabello, Emilio

Cabezas Sánchez, César

Cabrera, Rufino

Cáceres Carrera, Lorenzo

Campos, Miguel
Pontificia Universidad Católica de Chile, Chile.

Dirección Nacional de Maternidad e Infancia, Ministerio de Salud de la Nación, Argentina. Research Center for Food and Development (CIAD), Mexico.

Instituto Nacional de Cancerología de Bogotá, Colombia.

Universidad de Carabobo, Venezuela

Universidad Peruana Cayetano Heredia, Perú.

Escuela de Psicología, Pontificia Universidad Católica de Chile, Chile.

Hospital Universitari del Sagrat Cor, Universitat de Barcelona, Barcelona, España.

Universidad Peruana Cayetano Heredia, Perú.

Universidad Autónoma de Madrid, España.

Instituto Nacional de Salud, Perú.

Centre de Salut Pública Castelló, España.

Hospital Clínico Universitario Virgen de la Arrixaca, España.

Universidad Politécnica de Madrid, España.

Instituto de Salud Carlos III, España.

Universidad Peruana Cayetano Heredia, Perú.

Universidad Autónoma de Yucatán, México.

Hospital Universitario de Valme, España.

Instituto Nacional de Salud, Perú.

Associate Professor of Medicine, Medical Director, Inpatient Dialysis Unit, UPMC-

Presbyterian Hospital, EE UU.

Universidad Peruana Cayetano Heredia, Perú.

Universidad CES, Colombia.

Universidad Autónoma de Madrid, España.

Universidad Nacional de Rosario, Argentina.

Instituto Nacional de Salud, Perú.

Universidad Peruana Cayetano Heredia, Perú.

Universidad Peruana Cayetano Heredia, Perú.

Instituto Nacional de Salud, Perú.

Dirección General de Epidemiología, Ministerio de salud, Perú.

Instituto Conmemorativo Gorgas de Estudios de la Salud, Panamá.

Universidad Peruana Cayetano Heredia, Perú. 
Canal, Enrique

Canelo Aybar, Carlos

Carmona Fonseca, Jaime

Carrillo Inungaray, María Luisa

Carrión Chambilla, Jesús

Cartes Velásquez, Ricardo

Casavilca Zambrano, Sandro

Castaño Castrillón, José

Castillo, Estela

Catacora Villasante, Manuel

Chaidez Quiroz, Cristóbal

Chávez Jimeno, Helver

Chena Lilian,

Choperena, Gabriel

Coitinho Azevedo, Cecilia

Combariza Vallejo, Juan Felipe

Contreras Pulache, Hans

Correa Correa, Zamanda

Corti, Marcelo

Corvetto, Marcia

Crespo, María del Pilar

Culqui Lévano, Dante

de Kantor, Isabel N.

De la Cruz Aguiló, Rosario

De la Cruz, Walter

De la O Cavazos, Manuel

De Lima, Ana Rita

De los Santos, Maxy

Del Carmen Ortego Mate, María

Devera, Rodolfo

Díaz Cabezas, Ricardo

Díaz Franco, Asunción

Díaz Guzmán, Jaime

Díaz Vélez, Cristian

Divisón Garrote, Juan Antonio

Drago Serrano, María Elisa

Durá Ros, María Jesús

Durán, Pablo

Durante, Eduardo

Ecker, Lucie

Ecos, Rosa

Elizabeth Ferrer, Eladio

Español Cano, Santiago

Espinosa Larrañaga, Francisco

Espinoza Gonzalez, Ricardo

Fasciglione, María Paola

Felipe Tapias, Luis

Ferré, Tura

Ferrer Pérez, Victoria A.

Fica, Alberto

Fiestas Saldarriaga, Fabián

Figueiredo Ferraz, Hugo

Figueroa Pedraza, Dixis

Flores, Diana

Franco Ospina, Luis A.
US Naval Medical Research Unit-6, Perú.

Instituto Nacional de Salud, Perú.

Universidad de Antioquía, Colombia.

Universidad Autónoma de San Luis Potosí, México.

Hospital Nacional Arzobispo Loayza, Perú.

Universidad de Concepción, Chile.

Instituto Nacional de Enfermedades Neoplásicas, Perú.

Universidad de Manizales, Colombia.

Universidad de la República, Uruguay.

Hospital Hermilio Valdizan, Perú.

Centro de Investigación en Alimentación y Desarrollo, México.

Hospital Nacional Cayetano Heredia, Perú.

Universidad Nacional de Asunción, Paraguay.

Hospital Arantzazu, San Sebastián, España.

Ministerio de Salud Pública, Uruguay.

Instituto Nacional de Cancerología, Colombia.

Universidad Nacional Mayor de San Marcos, Perú.

Universidad del Cauca, Colombia.

Hospital de Enfermedades Infecciosas Francisco Javier Muñiz, Argentina.

Pontificia Universidad Católica de Chile, Chile.

Universidad Santiago de Cali, Colombia.

Instituto de Salud Carlos III, España.

Panel de Consultores en Tuberculosis, OMS, Argentina.

Hospital Clínico Universitario de Valencia, España.

Universidad Peruana Cayetano Heredia, Perú.

Hospital Universitario «Dr. José E. González», Universidad Autónoma de Nuevo León, México.

Facultad Ciencias de la Salud, Universidad de Carabobo, Venezuela.

US Naval Medical Research Unit-6, Perú.

Universidad de Cantabria, España.

Universidad de Oriente Núcleo Bolívar, Venezuela.

Facultad de Medicina, Universidad de Caldas, Colombia.

Instituto de Salud Carlos III, España.

Hospital Universitario "Doce de Octubre", España.

Hospital Almanzor Aguinaga Asenjo, Chiclayo, Perú.

Centro de Salud de Casas Ibáñez, España.

Universidad Autónoma Metropolitana, México.

Universidad de Cantabria, España.

Centro Latinoamericano de Perinatología OPS/OMS, Argentina.

Hospital Italiano de Buenos Aires, Argentina.

Instituto de Investigación de Nutricional, Perú.

Instituto Nacional de Ciencias Neurológicas, Perú.

Facultad de Ciencias de la Salud, Universidad de Carabobo, Venezuela.

Fundación Almadén "Francisco Javier de Villegas", España.

Centro Nacional de Investigación Documental en Salud, México.

Facultad de Medicina, Universidad de Los Andes, Chile.

Consejo Nacional de Investigaciones Científicas y Técnicas, Argentina.

Department of Surgery, Massachusetts General Hospital, Boston, EE UU.

Universitat Autónoma de Barcelona, España.

Facultad de Psicología, Universidad de las Islas Baleares, España.

Hospital Clínico Universidad de Chile.

Instituto Nacional de Salud, Perú.

Universidad de Valencia, España.

Universidade Estadual da Paraíba, Brasil.

Universidad Nacional Mayor de San Marcos, Perú.

Facultad de Ciencias Farmacéuticas, Universidad de Cartagena, Colombia. 
Gamboa, Dionisia

Gamendia Lorena, Fausto

García Bruce, Hernán

García Toro, Mauro

García, Herakles A.

Gianella Malca, Gonzalo Ernesto

Gioffré, Andrea

Girao, Italo

Gómez Huelgas, Ricardo

Gonzales Quispe, Isidro

Gonzales Rengifo, Gustavo

González Mariño, Mario A.

González, Horacio F.

Gual Sala, Arcadi

Guerra Allison, Humberto

Guerrero Pabon, Mario Francisco

Gutiérrez Ingunza, Ericson

Gutiérrez Villafuerte, César

Hami Sutton, Alicia

Hamui Sutton, Liz

Haro García, Luis

Heras Benito, Manuel

Hernández Díaz, Adrián V.

Hernandez Hernandez, Rosa

Hernández Hernández, Vladimir

Hernández Vásquez, Akram

Herrán Falla, Oscar Fernando

Herrera Calderón, Oscar

Horna Campos, Olivia

Hozbor, Daniela

Huamán Saavedra, Jorge

Huamaní Saldaña, Charles

Infante Campos, Alberto

Jarque, Isidro

Jiménez Mejías

Juárez Figueroa, Luis A.

Juárez García, Marcela

Juárez Ramírez, Clara

Justino Borges, Lucélia

Laguna Torres, Alberto

Lama Valdivia, Jaime

Lares Asseff, Ismael

Lewin, Sara

Liria Domínguez, María Reyna

Loayza Rodríguez, Luis

Loredo Abdalá, Arturo

Loret de Mola, Christian

Luaces Cubells, Carles

Maguiña Vargas, Ciro

Málaga Rodríguez, Germán

Marin Villa, Marcel

Márquez Hernández, Verónica

Marrodan Serrano, María Dolores
Universidad Peruana Cayetano Heredia, Perú.

Universidad Nacional Mayor de San Marcos, Perú.

Escuela de Medicina, Pontificia Universidad Católica de Chile, Chile.

Hospital Son Llàtzer, Palma de Mallorca, España.

Facultad de Ciencias Veterinarias, Universidad Central de Venezuela, Venezuela.

Clínica Ricardo Palma, Perú.

Instituto de Biotecnología, CICVyA, Argentina.

Universitat Pompeu Fabra, España

Sociedad Española de Medicina Interna, España.

Instituto de Ciencias Neurológicas, Perú.

Universidad Peruana Cayetano Heredia, Perú.

Universidad Nacional de Colombia, Colombia.

Instituto de Desarrollo e Investigaciones Pediátricas, Hospital de Niños de La Plata,

Argentina.

Facultad de Medicina, Universitat de Barcelona, España.

Universidad Peruana Cayetano Heredia, Perú.

Universidad Nacional de Colombia, Colombia.

Instituto Nacional de Salud, Perú.

Universidad Nacional Mayor de San Marcos, Perú.

Universidad Nacional Autónoma de México, México.

Universidad Nacional Autónoma de México.

Universidad Nacional Autónoma de México, México.

Hospital General de Segovia, España.

CNSP/INS, Lima, Perú.

Universidad Simón Bolívar, Venezuela.

Universidad Autónoma de Ciudad Juárez, México.

Universidad de Chile, Chile.

Universidad Industrial de Santander, Colombia.

Universidad Nacional San Luis Gonzaga, Ica, Perú.

Universidad Autónoma de Barcelona, España.

Universidad Nacional de La Plata, Argentina.

Universidad Nacional de Trujillo, Perú.

Instituto Nacional de Salud, Perú.

Escuela Nacional de Sanidad, Instituto de Salud Carlos III, España.

Hospital La Fe, España.

Facultad de Medicina, Universidad de Granada, España.

Clínica Condesa, Programa de VIH/SIDA, México.

Universidad Peruana Cayetano Heredia, Perú.

Instituto Nacional de Salud Pública, México.

Universidade Federal de Santa Catarina, Brasil.

US Naval Medical Research Unit-6, Perú.

Sociedad de Gerontología y Geriatría del Perú, Perú.

Centro Interdisciplinario de Investigación para el Desarrollo Integral Regional, CIIDIR-

IPN, México.

Universidad de la República, Uruguay.

Instituto de Investigación Nutricional, Perú.

US Naval Medical Research Unit-6, Perú

Comisión Coordinadora de los Institutos Nacionales de Salud y Hospitales de Alta

Especialidad de la Secretaría de Salud, México.

Universidad Peruana Cayetano Heredia, Perú.

Hospital Sant Joan de Déu, España.

Universidad Peruana Cayetano Heredia, Perú.

Universidad Peruana Cayetano Heredia, Perú.

Universidad de Antioquia, Colombia

Universidad de Almería, España.

Universidad Complutense, España. 
Martínez Fernández, Andrés

Máttar, Salim

Mayta Tristán, Percy

Mederos Cuervo, Lilian María

Mejía, Christian R.

Mendoza Arana, Pedro

Mendoza Ticona, Alberto

Merino Soto, Cesar A.

Mezones Holguín, Edward

Mini Díaz, Elsy

Miranda Montero, Jaime

Montaña Álvarez, Mariano

Montenegro Heredia, Sonia

Morales Briceño, Hugo

Moreno, Antonio

Morey Vargas, Oscar

Moriana Elvira, Juan Antonio

Morrison, Amy

Munayco, César

Mundo Rosas, Verónica

Murillo Palacios, Olga L.

Muskus, Carlos E.

Náquira Velarde, César Gabriel

Nara, Eva

Novik A., Victoria

Ordóñez Iriarte, José María

Ordoñez, Gonzalo A.

Ortega Valín, Luis

Osada Lily, Jorge

Oswaldo Jave, Héctor

Pajuelo Ramírez, Jaime

Palomino Salcedo, Miriam

Pamo Reyna, Oscar

Paredes Esparza, Rodolfo

Pareja Franco, René

Patiño Bedoya, Jair Alberto

Páz Díaz Camacho, Sylvia

Perales Cabrera, Alberto

Pereira, Claudio A.

Pita Martín De Portela, María Luz

Polanco Aguilar, Pablo

Poterico Rojas, Julio

Pretell Alva, E. Javier

Pulido Manzanero, José

Puray, Maritza

Quispe del Centro, Antonio M.

Ramírez, Augusto V.

Ramos Rodríguez, Mariana

Recuenco, Sergio

Rendón Macías, Mario Enrique

Requena Hernández, María del Carmen

Reyes López, Alfonso

Risco de Domínguez, Graciela

Risso, Gustavo

Rivero Martín, María José
Universidad Rey Juan Carlos, España.

Instituto de Investigaciones Biológicas del Trópico, Universidad de Córdoba, Colombia. Universidad Peruana de Ciencias Aplicadas, Perú.

Instituto de Medicina Tropical "Pedro Kouri", Cuba.

Universidad Peruana de Ciencias Aplicadas, Perú.

Universidad Nacional Mayor de San Marcos, Perú.

Instituto Nacional de Salud, Lima, Perú.

Universidad de San Martin de Porres, Perú.

Instituto Nacional de Salud, Perú.

Universidad Nacional Mayor de San Marcos, Perú.

Universidad Peruana Cayetano Heredia, Perú.

Centro Estatal del Adulto Mayor, México.

Facultad de Medicina, Universidad de Concepción, Chile.

Centro Médico Siglo XXI, México.

Grupo de Trabajo para el Estudio de los Brotes de Gastroenteritis Aguda Vírica en Cataluña, España.

Mayo Clinic, EE UU.

Universidad de Córdoba, España.

US Naval Medical Research Unit-6, Perú.

Dirección General de Epidemiología, Ministerio de Salud, Perú.

Instituto Nacional de Salud Pública, México.

Secretaria de Salud Pública Municipal de Cali, Colombia.

Universidad de Antioquia, Colombia

Universidad Nacional Mayor de San Marcos, Perú.

Instituto de Investigaciones en Ciencias de la Salud, Paraguay.

Universidad de Valparaíso, Chile.

Sociedad Española de Sanidad Ambiental, España.

Organización Panamericana de la Salud, Ecuador.

Farmacia y Hospital de León, Universidad de León, España.

Universidad Peruana Cayetano Heredia, Perú.

Ministerio de Salud, Perú.

Universidad Nacional Mayor de San Marcos, Perú.

Centro Nacional de Salud Pública, Instituto Nacional de Salud, Perú.

Universidad Peruana Cayetano Heredia, Lima, Perú.

Escuela de Medicina Veterinaria, Universidad Andrés Bello, Chile.

Instituto de Cancerología, Clínica Las Américas, Colombia.

Escuela de Microbiología, Universidad de Antioquía, Colombia.

Universidad Autónoma de Sinaloa, México.

Facultad de Medicina de la UNMSM, Lima, Perú.

Instituto de Investigaciones Médicas Alfredo Lanari, Argentina.

Universidad de Buenos Aires, Argentina.

Red Asistencial de Salud de Arequipa, Perú.

Universidad Peruana Cayetano Heredia, Perú.

Unidad de cysticercosis, Instituto de Ciencias Neurológicas, Perú.

Instituto de Salud Carlos III, España.

Universidad Nacional Mayor de San Marcos, Perú.

US Naval Medical Research Unit-6, Perú.

American College of Occupational and Environmental Medicine, Illinois, EE. UU.

US Naval Medical Research Unit-6, Perú.

Instituto Nacional de Salud, Perú.

Universidad La Salle, México.

Universidad de León, España.

Hospital Infantil de México "Federico Gómez", México.

Universidad Peruana de Ciencias Aplicadas, Perú.

Instituto de Investigaciones Cardiológicas "Prof. Dr. Alberto C. Taquini”, Argentina.

Hospital Universitario de Fuenlabrada, España. 
Rodríguez Amado, Jesús

Rodríguez Hernández, Jorge Martin

Rodríguez Morales, Alfonso

Rodríguez Rangel, Diego Andrés

Rodríguez Salvá, Armando

Rodríguez Villamizar, Laura

Rodríguez, Glacelidys

Rojas Cabrera, Ernesto

Rojas Vilca, José Luis

Rojas, Mario A.

Romaní Romaní, Franco

Romero Peñuela, Marlyn Hellen

Ron Guerrero, Carlos

Rosero del Grupo, Doris Amanda

Rotta Escalante, Roberto

Runzer Colmenares, Fernando

Russo, María Julieta

Salaverry García, Oswaldo

Sánchez Meca, Julio

Santarcángelo, Salomé

Schijman, Alejandro G.

Schilmann Halbinger, Astrid

Segura Cardona, Ángela María

Sena Barnabé, Anderson

Sesma Vázquez, Sergio

Solari Zerpa, Lely

Solis Cóndor, Risof

Solís García del Pozo, Julián

Soria de González Stojan, Analía

Soto Arquiñigo, Leslie

Soto Tarazona, Alonso

Suarez Cunza, Silvia

Suárez Moreno, Víctor

Tejada, Romina

Ticse Aguirre, Ray

Torres Ramírez, Luis

Traviezo Valles, Luis

Troncoso Corzo, Luzmila

Ugarte Gil, César Augusto

Valenzuela Rodríguez, Germán

Vargas Herrera, Javier

Vásquez, Fabián

Vega Dienstmaier, Johann

Vela Amieva, Marcela

Ventura Alfaro, Carmelita E.

Vera Gamboa, Ligia

Vidal Ledo, Maria

Villamonte Calanche, Wilfredo

Villena Chávez, Jaime

Vitriol Gaysinsky, Verónica

Zorrilla Torras, Belén
Pharmacy Department, Oriente University, Cuba.

Universidad Javeriana, Colombia.

Universidad Tecnológica de Pereira, Colombia.

Hospital de La Misericordia, Colombia.

Instituto Nacional de Higiene, Epidemiología y Microbiología, Cuba.

Facultad de Salud, Universidad Industrial de Santander, Colombia.

Universidad de Oriente, Venezuela.

Instituto de Investigaciones Biomédicas, Universidad Mayor de San Simón, Bolivia.

Universidad Peruana Cayetano Heredia, Perú.

Department of Pediatrics, Division of Neonatal-Perinatal Medicine, Wake Forest School of Medicine, EE UU.

Instituto Nacional de Salud, Perú.

Facultad de Ciencias Agropecuarias, Universidad de Caldas, Colombia.

Centro Estatal de Cancerología de Nayarit, México.

Escuela de Microbiología, Universidad de Antioquia, Colombia.

Hospital Nacional Profesor Dr. Alejandro Posadas, Argentina.

Centro Médico Naval, Perú

Departamento de Neurología Cognitiva, Fundación para la Lucha contra las Enfermedades Neurológicas de la Infancia, Argentina.

CENSI/INS, Lima, Perú.

Universidad de Murcia, España.

Hospital General de Niños Pedro de Elizalde, Argentina.

Instituto de Investigaciones en Ingeniería Genética y Biología Molecular, Argentina.

Instituto Nacional de Salud Pública, México.

Facultad de Medicina, Universidad CES, Colombia.

Universidad Nueve de Julio, Brasil.

Consejo de Coordinación para la Implementación del Sistema de Justicia Penal, Secretaría de Gobernación, México.

Instituto Nacional de Salud, Perú.

Sociedad Peruana de Neumología, Perú.

Hospital de Villarrobledo, España.

Facultad de Bioquímica, Química y Farmacia, Universidad Nacional de Tucumán, Argentina.

Universidad Peruana Cayetano Heredia, Perú.

Hospital Nacional Hipólito Unanue, Perú.

Universidad Nacional Mayor de San Marcos, Perú.

Instituto Nacional de Salud, Perú.

Instituto Nacional de Salud, Perú.

Universidad Peruana Cayetano Heredia, Perú.

Instituto Nacional de Ciencias Neurológicas, Perú.

Universidad Centroccidental Lisandro Alvarado, Venezuela.

Universidad Nacional Mayor de San Marcos, Perú.

Universidad Peruana Cayetano Heredia, Perú.

Clínica Médica Cayetano Heredia, Perú.

Instituto Nacional de Salud, Perú

Facultad de Medicina, Universidad de Chile, Chile.

Universidad Peruana Cayetano Heredia, Perú.

Instituto Nacional de Pediatría, México.

Instituto Nacional de Salud Pública, México.

Universidad Autónoma de Yucatán, México.

Escuela Nacional de Salud Pública La Habana, Cuba.

Centro de Investigación de Medicina Materno Fetal de Altura, Cusco, Perú.

Universidad Peruana Cayetano Heredia, Perú.

Unidad de Psiquiatría, Hospital de Curicó, Chile.

Dirección General de Atención Primaria, Consejería de Sanidad, España. 